\title{
Increasing Sensitivity and Selectivity of Gas Sensor- Systems by PLD-deposited Sensitive Layers and Micromachined Pre-Concentrators with MOF Layers
}

\author{
Andreas Schütze ${ }^{1}$, Tilman Sauerwald ${ }^{1}$, Martin Leidinger ${ }^{1}$, Joni Huotari ${ }^{2}$, Jyrki Lappalainen ${ }^{2}$, Max \\ Rieger $^{3}$, Jürgen Hürttlen ${ }^{3}$, Christine Alépée ${ }^{4}$, Jari Liimatainen ${ }^{5}$, Ville Kekkonen ${ }^{5}$ \\ ${ }^{1}$ Lab for Measurement Technology, Saarland University, Saarbruecken, Germany \\ ${ }^{2}$ Microelectronics and Materials Physics Laboratories, University of Oulu, Finland \\ ${ }^{3}$ Fraunhofer-Institute for Chemical Technologies ICT, Pfinztal, Germany \\ ${ }^{4}$ SGX Sensortech SA, Corcelles, Switzerland \\ ${ }^{5}$ Picodeon Ltd Oy, li, Finland \\ schuetze@LMT.uni-saarland.de
}

\begin{abstract}
The EU project SENSIndoor aims at the development of novel nanotechnology based intelligent sensor systems for selective monitoring of Volatile Organic Compounds (VOC) for demand controlled ventilation in indoor environments. Target gases are benzene, naphthalene and formaldehyde, as these VOCs are highly health relevant and occur in many indoor environments, thus making their monitoring a high priority for safe and healthy buildings. Relevant concentrations according to recommended values given by the $\mathrm{WHO}$ are in the low ppb range with benzene requiring the highest sensitivity to achieve a target concentration below $1 \mathrm{ppb}$. The SENSIndoor project makes use of two novel approaches to achieve this challenging goal: highly porous and well-controlled nano-scaled gassensitive layers deposited by Pulsed Laser Deposition (PLD) as well as selective pre-concentration using Metal-Organic Frameworks integrated on micro hotplates.
\end{abstract}

Key words: Indoor Air Quality, VOC detection, PLD-deposited layers, MOF-based pre-concentration

\section{Introduction}

SENSIndoor aims at the development of novel nanotechnology based intelligent sensor systems for selective monitoring of Volatile Organic Compounds (VOC) for demand controlled ventilation in indoor environments. Greatly reduced energy consumption without adverse health effects caused by the Sick Building Syndrome requires optimized ventilation schemes adapted to specific application scenarios like offices, hospitals, schools, nurseries or private homes.

Within the SENSIndoor project, novel sensor systems for extremely sensitive, highly selective and long-term stable operation will be studied and developed for advanced control of indoor air quality. The project makes use of both physical and chemical nanotechnologies for sensor components, MEMS technology for component realization and system integration as well as advanced signal processing and networking to integrate sensors into building control systems.

The foundations for the project were laid in the MNT-ERA.net project VOC-IDS (Volatile Organic Compound Indoor Discrimination Sensor) and the COST network EuNetAir. Within VOCIDS the most relevant target VOCs for indoor air quality monitoring as well as their target concentration limits were identified, the basis for integrating selective gas sensor systems in building automation was made by defining standards for KNX and Enocean networks and sensor systems based on conventional technologies were studied for selective detection of VOCs using temperature controlled operation (TCO) [1].

Within SENSIndoor Metal Oxide Semiconductor (MOS) and Gas-sensitive Field Effect Transistor (GasFET) sensors are studied as complementary sensor technologies, both based on proven microtechnologies to provide the basis for lowcost ubiquitous sensor systems. To achieve unrivalled sensitivity for hazardous indoor air pollutants, especially VOCs, novel nanocrystalline gas sensitive layer are studied, which are deposited using Pulsed Layer Deposition (PLD). The PLD technology is studied both at lab scale as well as in novel industrial scale processes to allow later scale-up of the deposition process. 
These sensor technologies are combined with gas pre-concentration based on MIPs (molecular imprinted polymers) and MOFs (metalorganic frameworks) to boost the sensitivity of the overall system. Dynamic operation of the gas sensor elements by temperature cycling combined with pattern recognition techniques is employed to further boost sensitivity and selectivity and expanded to optimally use the gas pre-concentration. The project thus combines physical and chemical nanotechnologies for extremely sensitive and selective gas sensing, MEMS technologies for low-power operation as well as low-cost manufacture and finally dynamic operating modes together with advanced signal processing for unrivalled system performance.

Sensor elements and systems are evaluated under controlled lab conditions derived from priority application scenarios. For this, a novel gas mixing system was previously developed in the VOC-IDS project allowing highly controlled measurements at sub-ppb concentrations and over a wide concentration range of more than 4 orders of magnitude for a single gas [2]. The performance of the new set-up was proven using GC-MS measurement.

The final demonstration of the SENSIndoor technology will include field tests with sensor systems integrated into building control systems. Fig. 1 provides an overview over the project; for further information refer to [3].

\section{PLD deposition of gas-sensitive layers}

Pulsed laser deposition is a very versatile method for depositing thin films and nanostructured layers from various materials, e.g. for gas sensing purposes. When using nanosecond laser PLD with a high oxygen partial pressure in the deposition chamber, nanoparticle formation starts during the deposition process leading to a highly porous nanostructured layer [4]. These types of layers are very suitable for gas sensing purposes because of their high specific surface area. Using this method, $\mathrm{WO}_{3}$ layers were deposited on MEMS micro hotplates to produce gas sensors with excellent selectivity vs. naphthalene [5].

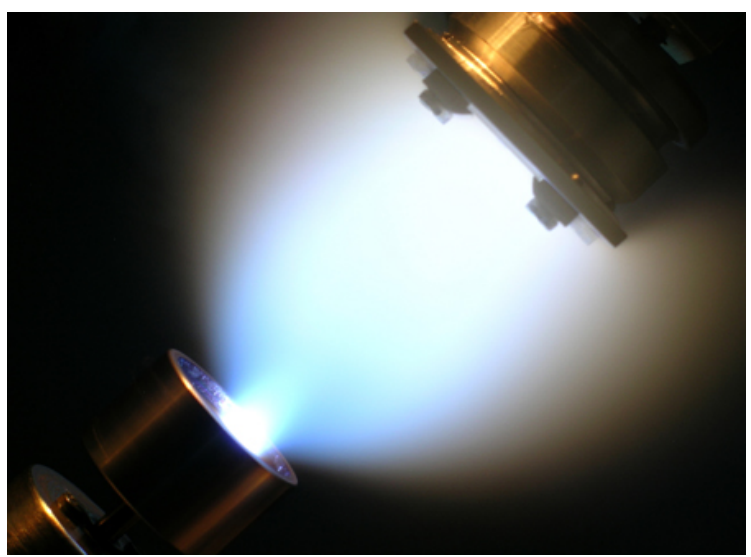

Fig. 2. Plasma resulting from high power nanosecond laser pulses focused on target (lower left) and resulting in coating of the substrate (upper right). Source: Oulu University, FETF.

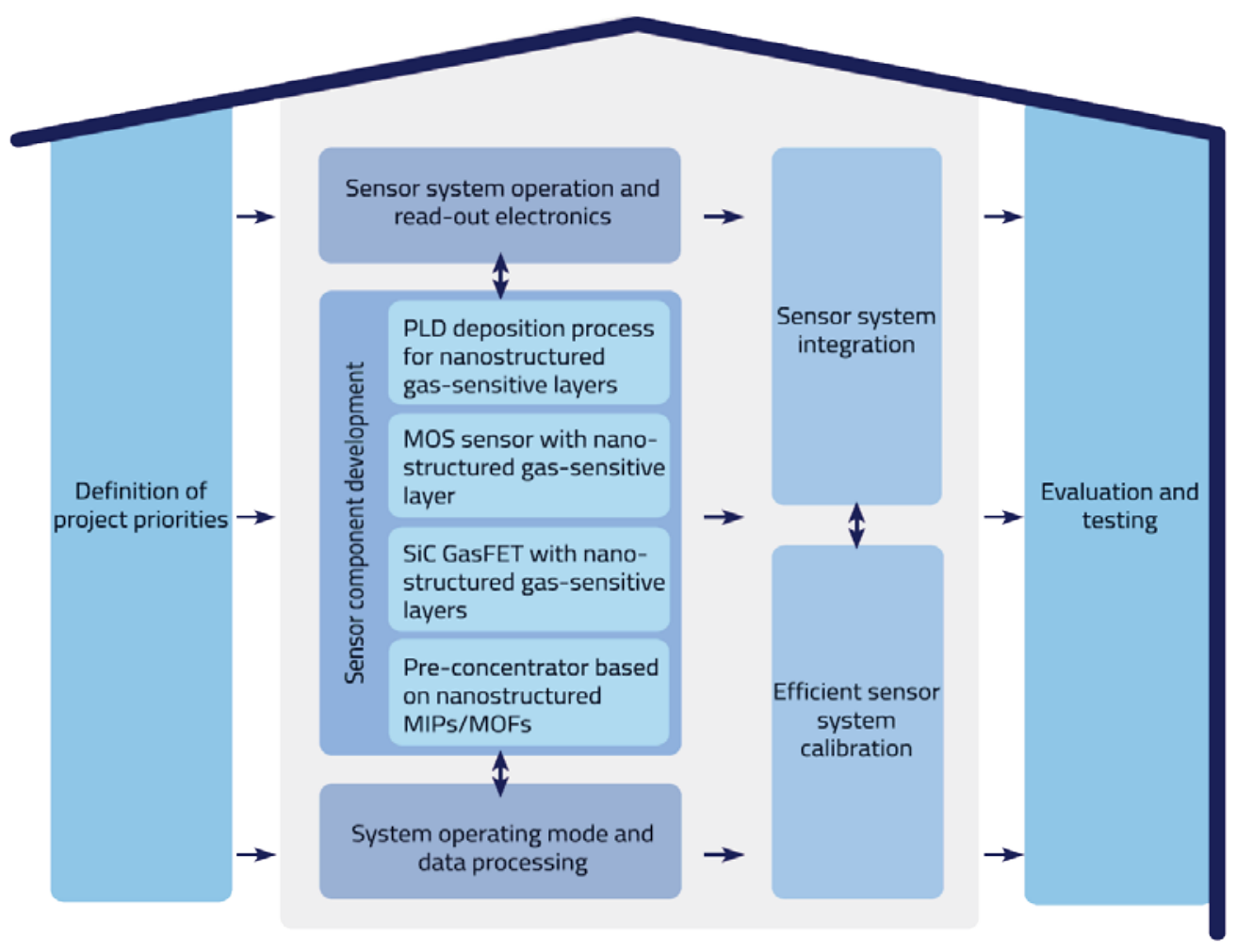

Fig. 1. Overview over the SENSIndoor project [3]. 
Currently, further experiments are performed using picosecond laser technology, allowing even further variation of process parameters and which is especially suitable for deposition of noble metals, e.g. to integrate catalyst materials like palladium or platinum in MOS sensors or to deposit nanoporous gate metallization for GasFET sensors. Both technologies have demonstrated the ability to detect VOCs in the required ppb concentration levels at least in laboratory environment [1, 6-8].

Preliminary results have shown that the sensor layers achieved with the PLD processes, Fig. 3, show excellent sensitivity comparable to the best conventional sensor layers. More importantly, the nanostructures are highly temperature stable thus allowing gas sensor operation at elevated temperatures. Further results concerning sensitivity selectivity and stability are currently prepared for publication [9].

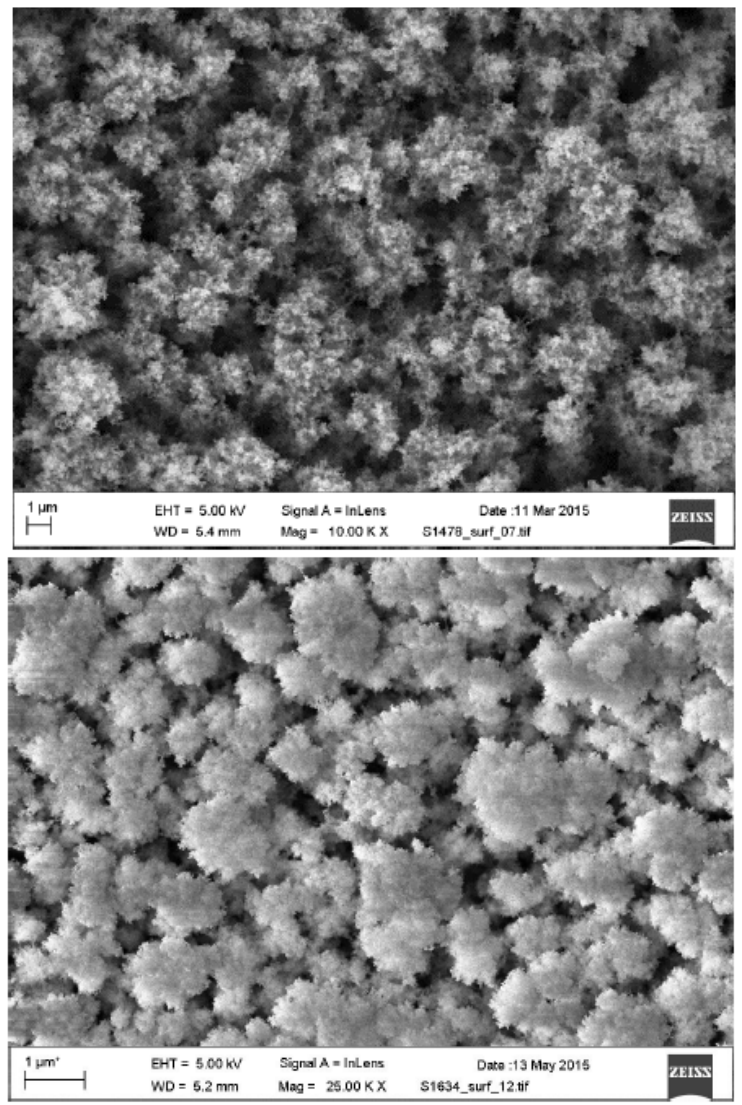

Fig. 3. Highly porous $\mathrm{WO}_{3}$ (top) and $\mathrm{SnO}_{2}$ (bottom) layers deposited by picosecond PLD [9].

\section{Boosting sensitivity and selectivity with MOF-based pre-concentration}

In order to detect low concentrations of VOCs, pre-concentration of the gases can be used. If selective absorption materials are used, these pre-concentrators can boost both sensitivity as well as selectivity of the overall system. Previously, pre-concentrators were mainly based on closed capillary columns requiring complex fabrication processes and also active pumping to achieve efficient transport of the desorbed gas to the detector $[10,11]$ thus leading to bulky and fairly costly sensor systems. We are developing a novel approach with adsorption materials integrated on MEMS micro-hotplates which are placed close to the detectors to allow passive transport of the desorbed gas molecules by diffusion only. Even though this approach is less efficient, we expect to achieve an improvement in sensitivity by at least one order of magnitude. This was already indicated in first experiments [12].

In our experiments, two different MOF materials (MIL-53 and HKUST-1) were deposited on alumina substrates with integrated heater structures to generate temperature controllable pre-concentrator devices. The platinum heater allows for active heating of the devices and thus controlled desorption of gases which were adsorbed on and in the MOF materials at low temperatures. MIL-53 is a three-dimensional metal-organic framework composed of aluminum metal-nodes and terephthalic acid molecules serving as linkers [13]. The structure itself bears channel-like structures with largest cavity diameter of about 7.3 to $7.7 \AA$. HKUST-1 (also often referred to as Cu-BTC) is a three dimensional metal-organic framework made up of $\mathrm{Cu}_{2}$ clusters and connecting trimesic-acid linkers [14]. The structure itself consists of three different interconnected pore sizes with $11 \AA$ being the largest ones. HKUST-1 bears so called open-metal sites that allow for Lewis bases (e.g. gaseous formaldehyde or ammonia) to coordinate.

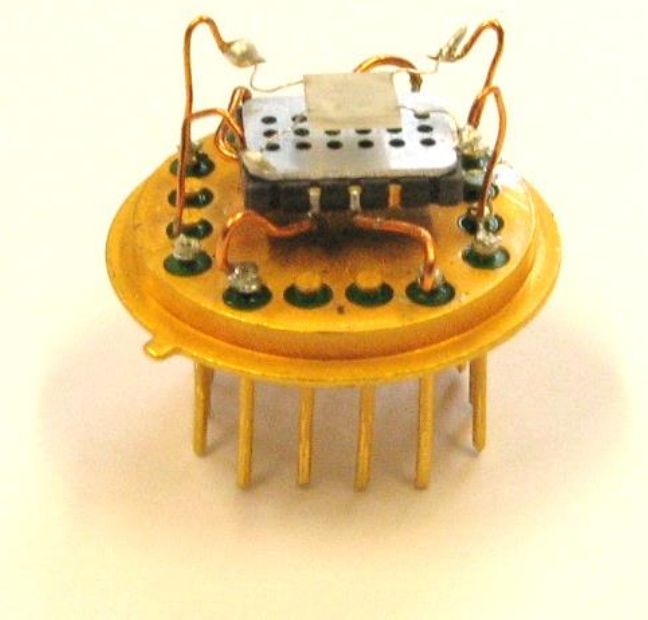

Fig. 4. First test set-up of the novel preconcentrator approach. A ceramic heater covered with the MOF material (top) is placed face-to-face of a standard micro gas-sensor. 
We are currently using multiphysics simulation to identify the best packaging concepts to combine gas sensor and pre-concentrator. These results have indicated that the gas pulses to be expected are on the order of a few seconds at most. Thus, this gas pulse has to be exactly synchronized with the temperature cycled operation of the gas sensors to achieve the desired functionality, i.e. the expected boost in sensitivity and selectivity.

\section{Conclusion and outlook}

The preliminary results achieved within the SENSIndoor project for the new PLD gassensitive layers and the novel pre-concentrator concept are very promising. Of course, to achieve the overall target of the project, many problems remain to be solved, e.g. selection of MOFs with high selectivity vs. the target gases, identification of a MOF deposition process suitable for mass production, selection of an efficient and low-cost packaging technology for combining pre-concentrators and sensor elements and definition of operating modes and signal evaluation algorithms to make full use of the potential provided by the new hardware.

We expect first fully integrated sensor systems with miniaturized electronics, adapted operating mode and suitable signal evaluation strategies within the next months. Integrated systems, developed jointly by the SENSIndoor partners, will be tested in lab and real operating environments. First test installations are planned for kindergartens and schools as children are highly vulnerable to air contamination.

\section{Acknowledgements}

The SENSIndoor project has received funding from the European Union's Seventh Framework Programme for research, technological development and demonstration under grant agreement No 604311.

The partners were brought together through the COST network TD1105 EuNetAir, which has also provided valuable input through many discussions with other network partners.

\section{References}

[1] M. Leidinger, T. Sauerwald, W. Reimringer, G. Ventura, and A. Schütze, Selective detection of hazardous VOCs for indoor air quality applications using a virtual gas sensor array, J. Sens. Sens. Syst., 3, 253-263 (2014), open access; doi: 10.5194/jsss-3-253-2014

[2] N. Helwig, M. Schüler, C. Bur, A. Schütze and T. Sauerwald, Gas mixing apparatus for automated gas sensor characterization, Meas. Sci. Technol. 25 (2014) 055903 (9pp); open access, doi: 10.1088/0957-0233/25/5/055903
[3] http://sensindoor.eu

[4] R.F. Wood, J.N. Leboeuf, K.R. Chen, D.B. Geohegan, A.A. Puretzky, Dynamics of Plume Propagation, Splitting, and Nanoparticle Formation During Pulsed-Laser Ablation, Applied Surface Science 127-129, 151-158 (1998); doi: 10.1016/S0169-4332(97)00625-9

[5] M. Leidinger, J. Huotari, T. Sauerwald, J. Lappalainen, A. Schütze, Nanostructured $\mathrm{WO}_{3}$ Semiconductor Gas Sensor for Selective Detection of Naphthalene, Proceedings SENSOR 2015, Nuremberg, May 19 - 21, 2015.

[6] M. Schüler, N. Helwig, G. Ventura, A. Schütze and T. Sauerwald, Detecting Trace-Level Concentrations of Volatile Organic Compounds with Metal Oxide Gas Sensors, Proceedings IEEE Sensors Conference 2013; Baltimore, MD, USA, Nov. 03 - 06, 2013.

[7] C. Bur, M. Andersson, A. Lloyd Spetz, A. Schütze, Detecting Volatile Organic Compounds in the ppb Range with Gas Sensitive Platinum gate SiC-Field Effect Transistors, IEEE Sensors Journal 14 (9), 3221 - 3228 (2014), doi: 10.1109/JSEN.2014.2326693

[8] D. Puglisi, J. Eriksson, C. Bur, A. Schuetze, A. Lloyd Spetz, M. Andersson, Catalytic metal-gate field effect transistors based on SiC for indoor air quality control, J. Sens. Sens. Syst., 4, 1-8 (2015), doi: 10.5194/jsss-4-1-2015

[9] V. Kekkonen, J. Liimatainen, S. Chaudhuri, T. Sauerwald, A. Schütze, Engineered metal and metal oxide gas sensor layers by pulsed laser deposition technology, IEEE Sensors Conference 2015; Busan, Korea, Nov. 2015, submitted.

[10] M. Kim, S. Mitra, A microfabricated microconcentrator for sensors and gas chromatography, J. Chromatogr. A, 996, 1-11 (2003), doi: 10.1016/S0021-9673(03)00560-0

[11] I. Gràcia, P. Ivanov, F. Blanco, N. Sabaté, X. Vilanova, X. Correig, L. Fonseca, E. Figueras, J. Santander, C. Cané, Sub-ppm gas sensor detection via spiral $\mu$-preconcentrator, Sens. Actuators B: Chem., 132, 149-154 (2008), doi: 10.1016/j.snb.2008.01.019

[12] M. Leidinger, M. Rieger, T. Sauerwald, M. Nägele, J. Hürttlen, A. Schütze, Trace gas VOC detection using metal-organic frameworks micro pre-concentrators and semiconductor gas sensors, EUROSENSORS 2015, Freiburg, Sept. 6-9, 2015, accepted.

[13] T. Loiseau, C. Serre, C. Huguenard, F. Taulelle, M. Henry, T. Bataille, G. Férey, A rationale for the large breathing of the porous aluminum terephthalate (MIL-53) upon hydration. Chemistry 10, 1373-1382 (2004), doi: 10.1002/chem.200305413

[14] S.S.Y. Chui, S.M.F. Lo, J.P.H. Charmant, A.G. Orpen, I.D. Williams, A chemically functionalizable nanoporous material [Cu3(TMA)2(H2O)3], Science 283, 1148-1150 (1999), doi: 10.1126/science.283.5405.1148 